\title{
Experimental research on single factor Enzymatic Extraction Polysaccharide barley
}

\author{
Yong-guang $\mathrm{Bi}^{1,2, \star}$, Yu-min $\mathrm{Li}^{2}$ \\ ${ }^{1}$ Guangxi Key Laboratory of Petrochemical Resource Processing and Process Intensification Technology, Guangxi University, Nanning \\ 530004, China \\ ${ }^{2}$ School of Pharmacy, Guangdong Pharmaceutical University, Guangzhou 510006, Guangdong, China
}

\begin{abstract}
This paper discusses the factors Enzymatic Extraction barley polysaccharides investigate the effect of hydrolysis time, hydrolysis temperature, enzyme dosage, solid-liquid ratio and $\mathrm{pH}$ value of the five factors of barley polysaccharide extraction rate, the results show : with the increase of hydrolysis time, pioneered polysaccharide extracted barley and then decreased. Hydrolysis of polysaccharides extraction rate over time and gradually increased with increasing temperature and the amount of enzyme hydrolysis, barley polysaccharide extraction rate is rising. With the rise within the experimental range of $\mathrm{PH}$ value, the extraction rate of polysaccharides of barley declined. With the increase of the solid-liquid ratio of polysaccharide extracted barley pioneered and then decreased.
\end{abstract}

Pale barley and sweet, slightly cold, favorable water swelling, spleen dewetting, getting in addition to weakness, heat pus and other effects, for the common benefits of water Shenshi medicine. (1) Anti-cancer: it contains selenium can inhibit cancer cell reproduction, can be used for Xiao cancer, adjuvant treatment of cervical cancer. Healthy people eat barley, make the body nimble, reducing the probability of cancer incidence. Spleen wet Sheng especially gastrointestinal cancer and lung phlegm dampness more appropriate. In Japan, the barley is seen as typical of the "anti-cancer food", but also to reduce the side effects of chemotherapy in cancer patients. (2) the spleen and stomach, promote metabolism: barley for containing a variety of vitamins and minerals, can promote metabolism and reduce the burden of gastrointestinal effects, as a disease or illness tonic food frail patients, regular consumption of barley foods on chronic enteritis, indigestion embolism also effective. (3) White skin: barley contains a certain amount of vitamin E, is a kind of beauty food, eat it regularly can keep delicate skin luster, eliminate acne, dark spots, improve the complexion. And it is caused by a viral infection for wart and so have some therapeutic effect. (4) Hair: nutritional hair, prevent hair loss and make hair smooth and soft effect. (5) Water Shenshi: favorable water swelling, spleen dewetting, getting in addition to weakness, heat pus and other effects, for the common benefits of water Shenshi medicine. (6) Prevention of beriberi: barley is rich in vitamin $\mathrm{B}$, it is very useful for prevention and treatment of beriberi $i^{[1]}$.

Raw barley cold side, diuresis Shenshi best line, can dehumidification in addition to wind, heat abscess, in addition to paralysis and pain of dysuria, edema, beriberi and rheumatism pain, the effect is significant.Fried barley is barley net take simmer fried to slightly yellow, when the muster remove and let cool slightly focal spot, slightly fragrant. In traditional Chinese medicine in the brewing method, there is a drug with wheat bran fry, namely fried barley bran. Heat pan first bran, an amount of one tenth of barley, heated to smoke, add the net barley, fry muster yellow when the surface was removed, after sieve and let cool to wheat bran, a slight aroma. This aroma depending on the degree of frying traditional Chinese medicine, is divided into fried yellow, Chaojiao and fried carbon. Fried yellow refers to simmer Scoop inherent aroma herbs, or muster, when burst for the degree. Fried yellow medicine can ease the over-bias, while distributing the aroma, as well as the role of qi stagnation.

People usually eat barley, mostly direct soup porridge. Can also use barley stew melon. Because the white gourd taste sweet light and cold, efficacy is known for the benefits of water swelling, treatment of edema and slimming Jiapin, and barley compatibility with food, like the physics of with resultant, a greater role. This soup for maximum effectiveness is dampness, swelling, weight loss, for symptoms of edema and wet body fat are applicable, also joint to limb pain, flexion stretch disadvantage and leucorrhea and opacity have certain curative effect. Winter cold, so when barley stew, must be added to dispel cold spring onion and ginger.

People can do it yourself cooked barley fried, can also go to the drugstore to buy. The common point is a mild, good jianpizhixie, is the treatment of spleen dampness diarrhea. Different is the dehumidifying effect than good fried barley bran fried, spleen dampness. Such as some people easy to diarrhea, to know the seed of job's tears can cure diarrhea due to spleen deficiency, often cooked barley soup drink, but could not see the effect, this is because the raw barley cooler side, if using roasted barley porridge or take a spoonful of tea to drink, the effect is very obvious. Stir fried with bran than fried barley and more prominent role.

This paper discusses the factors Enzymatic Extraction barley polysaccharide hydrolysis time to explore, hydrolysis temperature, enzyme dosage, solid-liquid ratio and $\mathrm{pH}$ value of the five factors on barley polysaccharide effect, provide a reference for industrial-scale production in accordance with.

\section{Materials and methods ${ }^{[2]}$}

\subsection{Raw materials and reagents}

Coix chinensis Tod. (Origin: Guizhou, specifications: 0.5kg/bag, Hubei Jingui Chinese Herbal Medicine Co., Ltd.). After sifted through a 40 mesh sieve to obtain honeysuckle powder and set aside. Glucose reference, Chinese medicines and biological products; phenol, 
concentrated sulfuric acid and deionized water, were of analytical grade.

\subsection{Instruments and Equipment}

H-6 constant temperature water bath (Jintan Honghua Instrument Factory); RE-52CS rotary evaporator (Gongyi City, the British valley to China Instrument Factory); I CHINABRAND circulating water pump (Gongyi City, the British valley to China instrument); UV1101 UV / Vis spectrophotometer (Shanghai Tian Mei scientific Instrument Co.); AY120 electronic analytical balance (Shimadzu Corporation).

1.3 Preparation of standard solutions of glucose ${ }^{[2]}$

1.4 Preparation of standard curve ${ }^{[2]}$

1.5 Determination of extraction yield of Coix chinensis $\operatorname{Tod}^{[2]}$.

\section{Results and analysis}

A change in a single factor, fixed remaining factors, the single factor experiment to study the effects of various factors on the extraction rate of polysaccharides barley.

\subsection{Examine the impact on the extraction rate of hydrolysis time}

In this study, barley flour (over 18 mesh sieve) $2.000 \mathrm{~g}$ as a raw material, reaction temperature $50{ }^{\circ} \mathrm{C}$, solid-liquid ratio 1:25 enzyme $0.016 \mathrm{~g}, \mathrm{PH}$ value of 5 to condition, reaction time was $30 \mathrm{~min}, 60 \mathrm{~min}$, $90 \mathrm{~min}, 120 \mathrm{~min}, 150 \mathrm{~min}$, $180 \mathrm{~min}$, extract the absorbance was measured in accordance with method 3.4, draw a graph (see Fig.1).

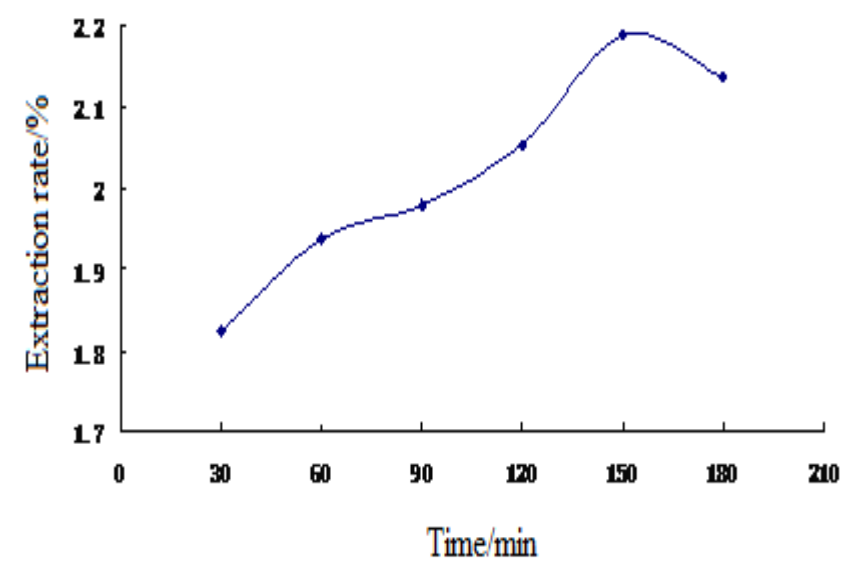

Fig.1 Hydrolysis time on the extraction rate influence graph

Different hydrolysis time corresponding extraction rate was $1.82 \%, 1.94 \%, 1.98 \%, 2.05 \%, 2.19 \%, 2.14 \%$. As can be seen from Fig.1, with the increase of hydrolysis time, pioneered polysaccharide extracted barley and then decreased. Hydrolysis of polysaccharides extraction rate over time and gradually increased to the highest in $150 \mathrm{~min}$, is because the longer the enzyme in contact with the cell wall, hydrolysis of the cell wall more thorough, more complete dissolution of the polysaccharide. But enzymes also have a certain polysaccharide degradation, when the hydrolysis time is too long, polysaccharide extraction rate but declined. Therefore, the hydrolysis time not too long.

\subsection{Examine the impact of temperature on the extraction rate of hydrolysis}

In this study, barley flour (over 18 mesh sieve) $2.000 \mathrm{~g}$ as a raw material, reaction time $60 \mathrm{~min}$, solid-liquid ratio $1: 25$, the amount of enzyme $0.016 \mathrm{~g}$, $\mathrm{PH}$ value of 5 for the condition, hydrolysis temperature of $30^{\circ} \mathrm{C}, 35^{\circ} \mathrm{C}, 40^{\circ} \mathrm{C}$, $45^{\circ} \mathrm{C}, 50^{\circ} \mathrm{C}, 55^{\circ} \mathrm{C} 60^{\circ} \mathrm{C}$ after pasting barley will affect the extraction rate), and the extract absorbance was measured in accordance with method 3.4, draw a graph (see Fig.2).

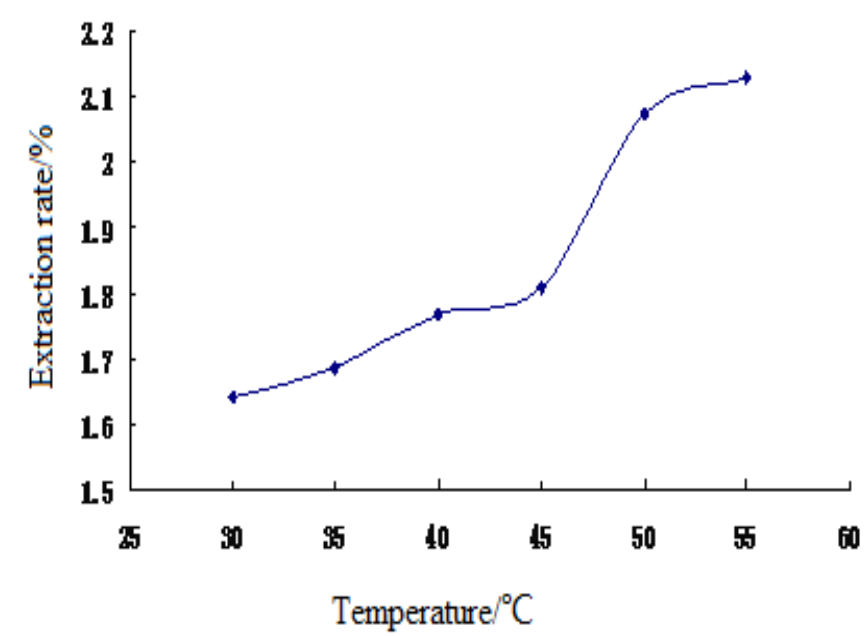

Fig.2 Hydrolysis temperature effect on the extraction rate graph

Different reaction temperature corresponding extraction rate was $1.64 \%, 1.69 \%, 1.77 \%, 1.81 \%, 2.07 \%$, $2.13 \%$. As can be seen from Fig.2, with the increase of reaction temperature, the extraction rate of polysaccharides barley rise. The higher the temperature, the average rate of movement of material elements increases, the greater the rate of diffusion; the same temperature for plant tissue infiltration and dissolution of the active ingredient is also promoted, so that under the action of cellulase, polysaccharides extraction rate extraction with rise of temperature rise.

\subsection{Investigate the amount of enzyme extraction rate}

In this study, barley flour (over 18 mesh sieve) $2.000 \mathrm{~g}$ as a raw material, reaction time $60 \mathrm{~min}$, reaction temperature $50{ }^{\circ} \mathrm{C}$, liquid ratio $1: 25$, $\mathrm{PH}$ value of 5 for the condition, the enzyme concentration was $0.004 \mathrm{~g}, 0.008 \mathrm{~g}, 0.012 \mathrm{~g}$, 0.016g, 0.020g, 0.024g, plotted in Figure (Fig.3). 


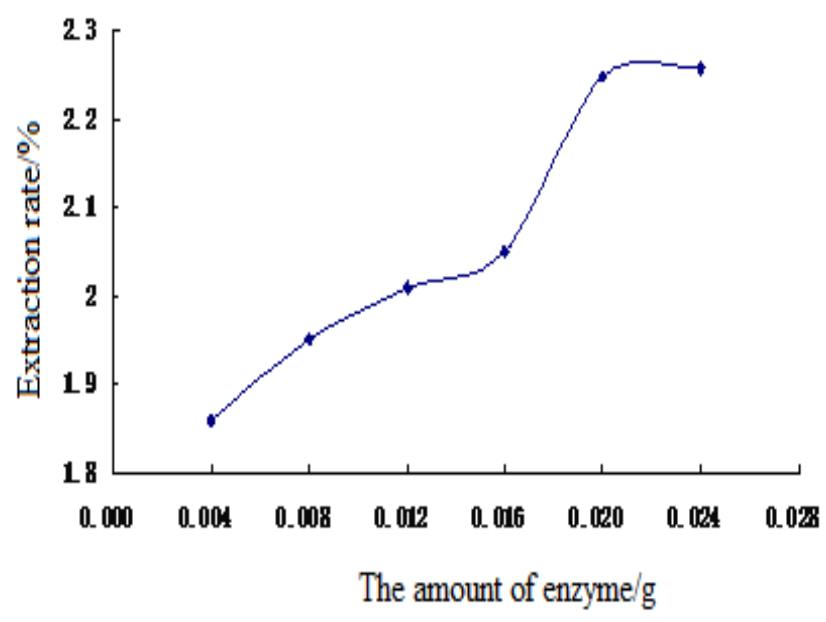

Fig.3 The amount of enzyme effect on the extraction rate graph

Different enzyme extract corresponding rates were $1.86 \%, 1.95 \%, 2.01 \%, 2.05 \%, 2.25 \%, 2.26 \%$. As can be seen from Fig.3, with the increase in the amount of enzyme extraction rate of polysaccharides of barley increased first steady trend. When small amount of enzyme, polysaccharide extraction rate rises slowly, because the enzyme only and no effect on other materials in contact with the portion of the feedstock; when the enzyme more full contact with the material, polysaccharide extraction rate increased significantly; then increase the amount of enzyme because the enzyme is saturated, so the polysaccharide extraction rate leveled off.

\subsection{The study on the extraction rate of $\mathrm{PH}$}

In this study, barley flour (over 18 mesh sieve) 2.000g as a raw material, reaction time $60 \mathrm{~min}$, reaction temperature $50{ }^{\circ} \mathrm{C}$, solid-liquid ratio $1: 25$, the amount of enzyme $0.016 \mathrm{~g}$ of conditions, $\mathrm{PH}$ value of $3,4,5,6,7,8$, the absorbance of the extract was measured according to the method of 3.4, to draw a graph (see Fig.4).

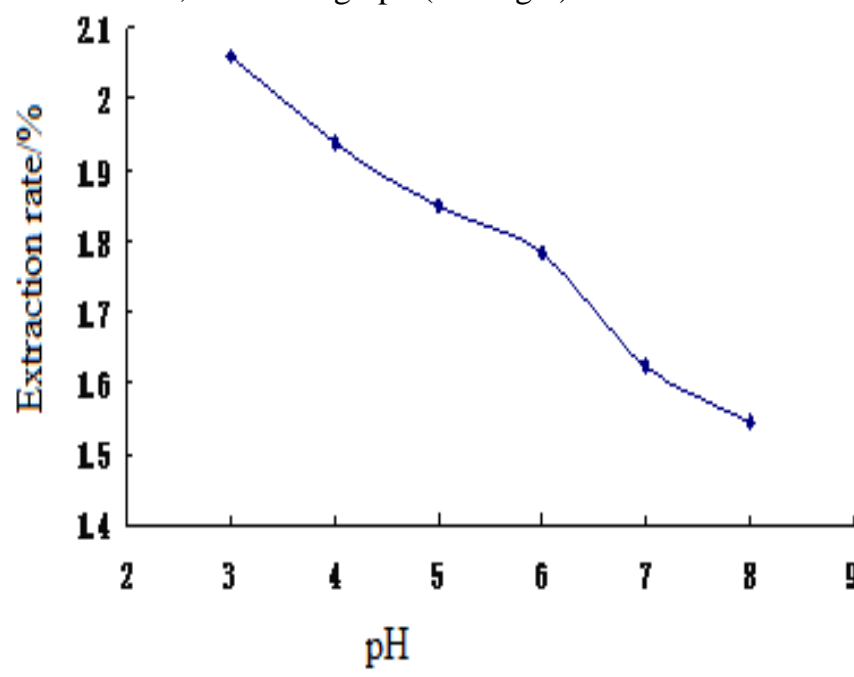

Fig.4 PH value impact on the extraction rate graph

$\mathrm{PH}$ values correspond to different extraction rates were $2.06 \%, 1.93 \%, 1.85 \%, 1.78 \%, 1.62 \%, 1.55 \%$. As can be seen from Fig.4, with the rise of $\mathrm{PH}$ values within the experimental range, the extraction rate of polysaccharides of barley declined. The reason $\mathrm{PH}$ enzymatic reactions affect many aspects, such as excess acid or alkali space allows enzyme conformational change in the enzyme inactivation. Another example $\mathrm{PH}$ substrate solution can be changed from the state, which affect binding to the enzyme. PH can affect some of the solutions on the enzyme molecule amino acid side chains from the state, especially the solution side groups enzymatic activity required from the state. $\mathrm{PH}$ values within the experimental range, with the $\mathrm{PH}$ value rises to the substrate dissociation deeper level, high $\mathrm{PH}$ further cause a certain degree of enzyme inactivation. Therefore, the PH polysaccharide extracted barley should be controlled within the range of low $\mathrm{PH}$, is considered the economic benefits, can be controlled at around $4 \mathrm{PH}$ value.

\subsection{Examine the impact of solid-liquid ratio on the extraction rate}

In this study, barley flour (over 18 mesh sieve) $2.000 \mathrm{~g}$ as a raw material, reaction time $60 \mathrm{~min}$, reaction temperature $50^{\circ} \mathrm{C}$, enzyme $0.016 \mathrm{~g}$, $\mathrm{PH}$ value of 5 for the condition, solid-liquid ratio of 1 : 10,1: 20, 1: $30,1: 40,1: 50,1: 60$, the absorbance of the extract was measured according to method 3.4, draw a graph (Fig.5).

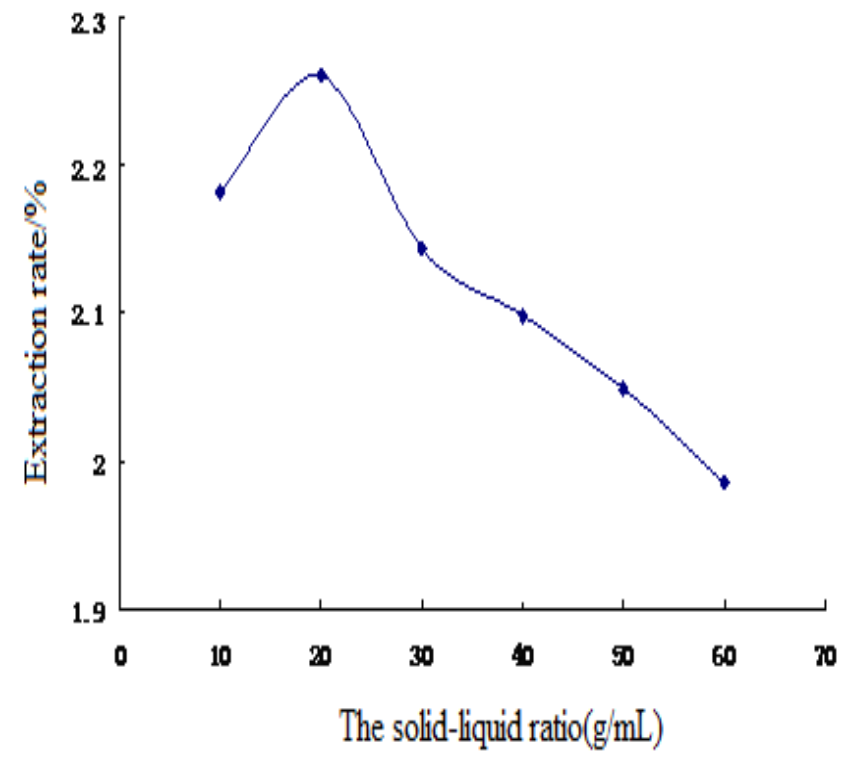

Fig.5 Liquid ratio on the extraction rate influence graph

Different than the corresponding solid-liquid extraction rates were $2.18 \%, 2.22 \%, 2.14 \%, 2.10 \%$, $2.05 \%, 1.99 \%$. As it can be seen from Figure 5, with the increase of solid-liquid ratio of polysaccharide extracted barley pioneered and then decreased. When less solvent, as a solvent and raw material not fully accessible and the enzyme has not fully in contact with the raw material, the dissolution of the polysaccharide is also easy to reach saturation, so the extraction rate is low; when the solvent is increased to make up for the shortcomings of less solvent, Therefore, the dissolution of polysaccharide increased; but when the solvent continues to increase, the enzyme is diluted and raw materials hit pong reduction, it 
polysaccharide extraction rate decreased with increasing solvent.

\section{Conclusion}

With the increase of hydrolysis time, pioneered polysaccharide extracted barley and then decreased. Hydrolysis of polysaccharides extraction rate over time and gradually increased with increasing temperature and the amount of enzyme hydrolysis, barley polysaccharide extraction rate is rising. With the rise within the experimental range of $\mathrm{PH}$ value, the extraction rate of polysaccharides of barley declined. With the increase of the solid-liquid ratio of polysaccharide extracted barley pioneered and then decreased.

\section{Acknowledgements}

This work was financially Supported by the Dean Project of Guangxi Key Laboratory of Petrochemical Resource Processing and Process Intensification Technology(2012K09)

\section{References}

[1] Mulloy B. The specificity of interactions between proteins and sulfated polysaccharides[J]. An Acad Bras Cienc, 2005,77(4): 651-664

[2] Yong-guang Bi, Yu-min Li. Study on the ultrasonic extraction of polysaccharides of barley rice[J]. Applied Mechanics and Materials, 2014, (513-517):332-335. 